Kragujevac Journal of Mathematics

Volume 45(5) (2021), Pages 787-796.

\title{
BOUNDEDNESS OF CERTAIN SYSTEM OF SECOND ORDER DIFFERENTIAL EQUATIONS
}

\author{
M. O. OMEIKE ${ }^{1}$, A. A. ADEYANJU' 1 , D. O. ADAMS ${ }^{1}$, AND A. L. OLUTIMO ${ }^{2}$ \\ ABSTRACT. This work is concerned with the ultimate boundedness of solutions of \\ the system of vector differential equations

$$
\dot{X}=H(Y), \quad \dot{Y}=-F(X, Y) Y-G(X)+P(t, X, Y),
$$ \\ where $t \in \mathbb{R}^{+}, X=X(t), Y=Y(t) \in \mathbb{R}^{n}, F: \mathbb{R}^{n} \times \mathbb{R}^{n} \rightarrow \mathbb{R}^{n \times n}, G, H: \mathbb{R}^{n} \rightarrow$ \\ $\mathbb{R}^{n}$ and $P: \mathbb{R}^{+} \times \mathbb{R}^{n} \times \mathbb{R}^{n} \rightarrow \mathbb{R}^{n}$. By using a Lyapunov function as a basic technique, \\ we prove that the solutions of the system of equations are ultimately bounded. In \\ addition, result obtained includes and improves some related results in literature.
}

\section{INTRODUCTION}

For over five decades, many authors have dealt considerably with qualitative properties of solutions (namely, stability, boundedness, convergence, existence of periodic solutions) of first order and higher order ordinary differential equations using the direct method of Lyapunov (also known as the second method of Lyapunov) [1-16]. This method enables us to determine the qualitative properties of solutions of a differential equation without actually finding its analytic solution. The method entails the construction of a positive definite function, whose derivative with respect to $t$ along the solution path is negative semi-definite. However, the construction of this function remains a general problem [10].

Using the Lyapunov's direct method, many authors have obtained boundedness results of solutions of scalar differential equations $[1,4,9-11,14,16]$, and some others have extended these results to vector differential equations $[2,3,5,7,8,12,13,15]$.

Key words and phrases. Boundedness, Lyapunov function, differential equations of second order. 2010 Mathematics Subject Classification. Primary: 34C11.

DOI 10.46793/KgJMat2105.787O

Received: March 28, 2018.

Accepted: May 21, 2019. 
Recently, Omeike et al. [8] considered the system of equations

$$
\dot{X}=Y, \quad \dot{Y}=-F(X, Y) Y-G(X)+P(t, X, Y),
$$

where $X, Y: \mathbb{R}^{+} \rightarrow \mathbb{R}^{n}, G: \mathbb{R}^{n} \rightarrow \mathbb{R}^{n}, P: \mathbb{R}^{+} \times \mathbb{R}^{n} \times \mathbb{R}^{n} \rightarrow \mathbb{R}^{n}, F$ is an $n \times n$ continuous symmetric positive definite matrix function for the arguments displayed explicitly, $\mathbb{R}$ denotes the real line, $-\infty<t<\infty, \mathbb{R}^{n}$ denotes the real $n$-dimensional Euclidean space equipped with the usual norm $\|\cdot\|$, and the dots (which appear in the (1.1)) as usual indicate differentiation with respect to $t$. (1.1) is a system derivable from the second order equation

$$
\ddot{X}+F(X, \dot{X}) \dot{X}+G(X)=P(t, X, \dot{X}),
$$

by setting $\dot{X}=Y$. (1.1) is an $n$-dimensional analogue of a system of equation

$$
\begin{aligned}
& \dot{x}=y, \\
& \dot{y}=-f(x, y) y-g(x)+p(t, x, y),
\end{aligned}
$$

studied by Tejumola [11], an equation of motion in Mathematical Physics. Omeike et al. [8] extended the results obtained in Tejumola [11] to (1.1) and obtained conditions which guarantee boundedness of solutions. Tejumola [12] further studied (1.2) in the form

$$
\begin{aligned}
& \dot{x}=h(y), \\
& \dot{y}=-f(x, y) y-g(x)+p(t, x, y),
\end{aligned}
$$

for boundedness of solutions. By constructing an incomplete Lyapunov function (see E. N. Chukwu [4]) and augmenting with a signum function a boundedness result was proved. In this present work, we extend the result obtained by Tejumola [12] to the $n$-dimensional analogue of (1.3), given by

$$
\begin{aligned}
& \dot{X}=H(Y), \\
& \dot{Y}=-F(X, Y) Y-G(X)+P(t, X, Y),
\end{aligned}
$$

where $H: \mathbb{R}^{n} \rightarrow \mathbb{R}^{n}$ and $X, Y, F, G$ and $P$ are as described above. It is also assumed that $F, G, H$ and $P$ are continuous for the argument displayed explicitly. In addition, the existence and uniqueness of the solutions of (1.4) with any prescribed initial conditions will be assumed (see Picard-Lindelof theorem in [9]).

The motivation for the present work is derived from the works of Tejumola $[11,12]$ and Omeike et al. [8]. We prove that solutions of (1.4) are bounded. To the best of our knowledge, no author in the literature has extended the boundedness result obtained by Tejumola [12] to (1.4).

\section{Notations}

We shall use the notation as given in [2]. Throughout this paper $\delta$ 's, $\Delta$ 's and $D$ 's with or without suffixes will denote positive constants whose magnitudes depend on an $n \times n$ matrix function $F(X, Y)$ and vector functions $H(Y), P(t, X, Y)$. The $\delta$ 's, 
$\Delta$ 's and $D$ 's with numerical or alphabetical suffixes shall retain fixed magnitudes, while those without suffixes are not necessarily the same at each occurrence.

Also, we shall denote the scalar product $\langle X, Y\rangle$ of any vectors $X, Y$ in $\mathbb{R}^{n}$, with respective components $\left(x_{1}, x_{2}, \ldots, x_{n}\right)$ and $\left(y_{1}, y_{2}, \ldots, y_{n}\right)$ by $\sum_{i=1}^{n} x_{i} y_{i}$. In particular, $\langle X, X\rangle=\|X\|^{2}$. Finally, by $\operatorname{sgn} X$, we mean $\left(\operatorname{sgn} x_{1}, \operatorname{sgn} x_{2}, \ldots, \operatorname{sgn} x_{n}\right), x_{i} \neq 0$, and $\|\operatorname{sgn} X\|=\sqrt{n}>0$.

\section{Main Results}

The following algebraic results will be required in the proofs of our main results.

Lemma 3.1. Let $A$ be a real symmetric positive definite $n \times n$ matrix. Then for $X \in \mathbb{R}^{n}, \delta_{a}\|X\|^{2} \leq\langle A X, X\rangle \leq \Delta_{a}\|X\|^{2}$, where $\delta_{a}$ and $\Delta_{a}$ are, respectively, the least and greatest eigenvalues of the matrix $A$.

Proof. See $[6,13]$.

Lemma 3.2. Let $G(0)=0=H(0)$ and assume that the matrices $A, J_{g}(X)$ and $J_{h}(Y)$ are symmetric, positive definite and commute pairwise for all $X, Y \in \mathbb{R}^{n}$. Then

$$
\begin{aligned}
\langle G(X), A X\rangle & =\int_{0}^{1} X^{T} A J_{g}(\sigma X) X d \sigma, \\
\langle H(Y), A Y\rangle & =\int_{0}^{1} Y^{T} A J_{h}(\sigma Y) Y d \sigma,
\end{aligned}
$$

where $J_{g}(X)$ and $J_{h}(Y)$ are respectively the Jacobian matrices $\frac{\partial g_{i}}{\partial x_{j}}$ and $\frac{\partial h_{i}}{\partial y_{j}}$ of $G(X)$ and $H(Y)$.

Proof. See $[5,13]$.

Lemma 3.3. Let $G(0)=0$ and assume that $J_{g}(X)$ is symmetric for all arbitrary $X \in \mathbb{R}^{n}$. Then

for all $X=X(t) \in \mathbb{R}^{n}$.

$$
\frac{d}{d t} \int_{0}^{1}\langle G(\sigma X), X\rangle d \sigma=\langle G(X), \dot{X}\rangle
$$

Proof. See [5].

Our main theorems are the following.

Theorem 3.1. Let $a, L, \beta, \Delta_{f}, \Delta_{g}, \Delta_{h}, \delta_{f}, \delta_{g}, \delta_{h}$ be positive constants and let all the basic assumptions imposed on $F, G, H$ and $P$ hold, and that $G(0)=H(0)=0$ hold. Suppose further that for any arbitrary $X, Y \in \mathbb{R}^{n}$

(i) $J_{g}(X), J_{h}(Y)$ are symmetric and positive definite;

(ii) the eigenvalues $\lambda_{i}(F(X, Y)), \lambda_{i}\left(J_{g}(X)\right), \lambda_{i}\left(J_{h}(Y)\right)$ of $F(X, Y), J_{g}(X)$ and $J_{h}(Y)$ respectively satisfy

$$
\begin{aligned}
& 0<\delta_{f} \leq \lambda_{i}(F(X, Y)) \leq \Delta_{f}, \\
& 0<\delta_{g} \leq \lambda_{i}\left(J_{g}(X)\right) \leq \Delta_{g}
\end{aligned}
$$




$$
0<\delta_{h} \leq \lambda_{i}\left(J_{h}(Y)\right) \leq \Delta_{h}
$$

(iii)

$$
\|P(t, X, Y)\| \leq a
$$

where $a$ is a positive constant.

Suppose further that

$$
\alpha\langle G(X), \operatorname{sgn} X\rangle \rightarrow \infty \quad \text { as } \quad\|X\| \rightarrow \infty,
$$

where $\alpha=\operatorname{sgn}\langle G(X), \operatorname{sgn} X\rangle$.

Then there exists a finite constant $K$ whose magnitude depends only on the constants $a, L, \beta, \Delta_{f}, \Delta_{g}, \Delta_{h}, \delta_{f}, \delta_{g}, \delta_{h}$, as well as the function $G(X)$ such that every solution $(X(t), Y(t))$ of (1.4) ultimately satisfies

$$
\|X(t)\| \leq K, \quad\|Y(t)\| \leq K .
$$

Theorem 3.2. In addition to the conditions (i) and (ii) of Theorem 3.1, suppose

(i) for all $t, X$ and $Y$

$$
\|P(t, X, Y)\| \leq \mu\|Y\|, \quad \mu>0,
$$

and

(ii)

$$
\lim _{\|X\| \rightarrow \infty} \alpha\langle G(X), \operatorname{sgn} X\rangle \rightarrow \infty .
$$

Then there exists a finite positive constant $K$ whose magnitude depends only on the constants a, $L, \beta, \mu, \Delta_{f}, \Delta_{g}, \Delta_{h}, \delta_{f}, \delta_{g}, \delta_{h}$ as well as the function $G(X), H(Y)$ such that every solution $(X(t), Y(t))$ of (1.4) ultimately satisfies (3.6).

\section{Proof of Main Results and Example}

Proof of Theorem 3.1. Our method of proof, which makes use of the adaptation of the well-known Yoshizawa [16] technique, is the same as in [8].

Let the continuous function $U=U(X, Y)$ be defined by

$$
U=U_{1}+U_{2}+1
$$

where

$$
\begin{gathered}
U_{1}=\int_{0}^{1}\langle H(\sigma Y), Y\rangle d \sigma+\int_{0}^{1}\langle G(\sigma X), X\rangle d \sigma \\
U_{2}=\left\{\begin{array}{ll}
\frac{L^{-1}}{\sqrt{n}} \alpha\langle Y, \operatorname{sgn} X\rangle, & \|Y\| \leq L, \\
\frac{1}{n}\langle\operatorname{sgn} X, \operatorname{sgn} Y\rangle, & \|Y\| \geq L,
\end{array} \quad \text { if }\|X\| \geq 1,\right.
\end{gathered}
$$


or

$$
U_{2}=\left\{\begin{array}{ll}
L^{-1}\langle X, Y\rangle, & \|Y\| \leq L, \\
\frac{1}{\sqrt{n}}\langle X, \operatorname{sgn} Y\rangle, & \|Y\| \geq L,
\end{array} \quad \text { if }\|X\| \leq 1 .\right.
$$

We shall show that $U(X, Y)$ satisfies

$$
U(X, Y) \rightarrow+\infty \text { as }\|X\|^{2}+\|Y\|^{2} \rightarrow+\infty .
$$

From the definition of $U_{2}$, we can show that $\left|U_{2}\right| \leq 1$ as follows.

If $\|X\| \geq 1$, we obtain

$$
\begin{aligned}
& \left|U_{2}\right|= \begin{cases}\left|\frac{L^{-1}}{\sqrt{n}} \alpha\langle Y, \operatorname{sgn} X\rangle\right|, & \|Y\| \leq L, \\
\left|\frac{1}{n}\langle\operatorname{sgn} X, \operatorname{sgn} Y\rangle\right|, \quad\|Y\| \geq L, & \text { if }\|X\| \geq 1,\end{cases} \\
& \leq\left\{\begin{array}{ll}
\frac{L^{-1}}{\sqrt{n}}|\langle Y, \operatorname{sgn} X\rangle|, & \|Y\| \leq L, \\
\frac{1}{n}|\langle\operatorname{sgn} X, \operatorname{sgn} Y\rangle|, & \|Y\| \geq L,
\end{array} \quad \text { if }\|X\| \geq 1,\right. \\
& \leq\left\{\begin{array}{ll}
\frac{L^{-1}}{\sqrt{n}}\|Y\|\|\operatorname{sgn} X\|, & \|Y\| \leq L, \\
\frac{1}{n}\|\operatorname{sgn} X\|\|\operatorname{sgn} Y\|, & \|Y\| \geq L,
\end{array} \quad \text { if }\|X\| \geq 1,\right. \\
& \leq\left\{\begin{array}{ll}
\frac{L^{-1}}{\sqrt{n}} \times L \times \sqrt{n}=1, & \|Y\| \leq L, \\
\frac{1}{n} \times \sqrt{n} \times \sqrt{n}=1, & \|Y\| \geq L,
\end{array} \quad \text { if }\|X\| \geq 1 .\right.
\end{aligned}
$$

Similarly, if $\|X\| \leq 1$, we obtain

$$
\begin{aligned}
& \left|U_{2}\right|=\left\{\begin{array}{ll}
\left|L^{-1}\langle X, Y\rangle\right|, & \|Y\| \leq L, \\
\left|\frac{1}{\sqrt{n}}\langle X, \operatorname{sgn} Y\rangle\right|, & \|Y\| \geq L,
\end{array} \quad \text { if }\|X\| \leq 1,\right. \\
& \leq\left\{\begin{array}{ll}
L^{-1}|\langle X, Y\rangle|, & \|Y\| \leq L, \\
\frac{1}{\sqrt{n}}|\langle X, \operatorname{sgn} Y\rangle|, & \|Y\| \geq L,
\end{array} \quad \text { if }\|X\| \leq 1,\right. \\
& \leq\left\{\begin{array}{ll}
L^{-1}\|X\|\|Y\|, & \|Y\| \leq L, \\
\frac{1}{\sqrt{n}}\|X\|\|\operatorname{sgn} Y\|, & \|Y\| \geq L,
\end{array} \quad \text { if }\|X\| \leq 1,\right. \\
& \leq\left\{\begin{array}{l}
L^{-1} \times 1 \times L=1, \quad\|Y\| \leq L, \\
\frac{1}{\sqrt{n}} \times 1 \times \sqrt{n}=1, \quad\|Y\| \geq L, \quad \text { if }\|X\| \leq 1 .
\end{array}\right.
\end{aligned}
$$

Thus, we have $\left|U_{2}\right| \leq 1$. 
Now, since $\left|U_{2}\right| \leq 1$, (4.1) yields $U \geq U_{1}$, and by Lemma 3.2, followed by Lemma 3.1 and inequalities (3.2) and (3.3), we have

$$
U_{1} \geq D_{0}\left(\|X\|^{2}+\|Y\|^{2}\right)
$$

where $D_{0}=\min \left\{\delta_{h}, \delta_{g}\right\}$. Thus,

$$
U(X, Y) \rightarrow \infty \quad \text { as } \quad\|X\|^{2}+\|Y\|^{2} \rightarrow \infty .
$$

We are now left to show that $\dot{U}$ exists and that there are finite constants $D_{1}, D_{2}$ such that

$$
\dot{U} \leq-D_{1}, \quad \text { if }\|X\|^{2}+\|Y\|^{2} \geq D_{2}
$$

From this and (4.5) it will then follow, just as in [8], that there is a constant $D>0$ such that every solution $(X(t), Y(t))$ of (1.4) ultimately satisfies

$$
\|X\|^{2}+\|Y\|^{2} \leq D
$$

and this verifies (3.6).

To verify (4.7), observe from (4.1) to (4.4) and (1.4) that by applying Lemma 3.3 to $U_{1}$, we obtain

$$
\dot{U}=\dot{U}_{1}+\dot{U}_{2}
$$

where

$$
\dot{U}_{1}=-\langle H(Y), F(X, Y) Y\rangle+\langle H(Y), P(t, X, Y)\rangle
$$

and

$$
\dot{U}_{2}=\left\{\begin{array}{ll}
\frac{L^{-1}}{\sqrt{n}} \alpha\langle-F(X, Y) Y-G(X)+P(t, X, Y), \operatorname{sgn} X\rangle, & \|Y\| \leq L, \\
0, & \|Y\| \geq L,
\end{array} \quad \text { if }\|X\| \geq 1,\right.
$$

or

$$
\dot{U}_{2}= \begin{cases}L^{-1}\langle H(Y), Y\rangle+L^{-1}\langle-F(X, Y) Y-G(X)+P(t, X, Y), X\rangle, & \|Y\| \leq L, \\ \frac{1}{\sqrt{n}}\langle H(Y), \operatorname{sgn} Y\rangle, & \|Y\| \geq L,\end{cases}
$$

if $\|X\| \leq 1$. Thus, if $\|Y\| \leq L, \dot{U}_{2}$ satisfies

$$
\dot{U}_{2}=-\frac{\alpha}{L \sqrt{n}}\langle F(X, Y) Y, \operatorname{sgn} X\rangle-\frac{\alpha}{L \sqrt{n}}\langle G(X), \operatorname{sgn} X\rangle+\frac{\alpha}{L \sqrt{n}}\langle P(t, X, Y), \operatorname{sgn} X\rangle,
$$

if $\|X\| \geq 1$, or

(4.13) $\dot{U}_{2}=\frac{1}{L}\langle H(Y), Y\rangle-\langle X, F(X, Y) Y\rangle-\langle X, G(X)\rangle+\langle P(t, X, Y), X\rangle$ if $\|X\| \leq 1$. 
But if $\|Y\| \geq L$, then

$$
\dot{U}_{2}= \begin{cases}0, & \|X\| \geq 1 \\ \frac{1}{\sqrt{n}}\langle H(Y), \operatorname{sgn} Y\rangle, & \|X\| \leq 1\end{cases}
$$

In obtaining estimates for $\dot{U}$ we shall consider points outside of the closed bounded set defined by $\|X\| \leq 1$ and $\|Y\| \leq L$. It will be convenient to consider the following three regions in turn: (I) $\|X\| \geq 1$ and $\|Y\| \leq L$, (II) $\|X\| \leq 1$ and $\|Y\| \geq L$, and (III) $\|X\| \geq 1$ and $\|Y\| \geq L$. For the case (I), we have from (4.8), (4.9) and (4.12) that

$$
\begin{aligned}
\dot{U}= & -\langle H(Y), F(X, Y) Y\rangle+\langle H(Y), P(t, X, Y)\rangle-\frac{\alpha}{L \sqrt{n}}\langle F(X, Y) Y, \operatorname{sgn} X\rangle \\
& -\frac{\alpha}{L \sqrt{n}}\langle G(X), \operatorname{sgn} X\rangle+\frac{\alpha}{L \sqrt{n}}\langle P(t, X, Y), \operatorname{sgn} X\rangle,
\end{aligned}
$$

so that by (3.1)-(3.4), and setting $\beta=\sqrt{n}$,

$$
\dot{U} \leq-\frac{1}{L \beta}\left(\alpha\langle G(X), \operatorname{sgn} X\rangle-\beta a \Delta_{h} L^{2}\right)+\Delta_{f},
$$

since $\|Y\| \leq L$. Thus, in view of $(3.5)$, there exists a finite constant $D_{3}(>1)$, sufficiently large, such that

$$
\dot{U} \leq-1 \text { provided }\|X\| \geq D_{3} .
$$

As for the case (II): $\|X\| \leq 1$ and $\|Y\| \geq L$, we have from (4.8), (4.9) and (4.14) that

$$
\dot{U}=-\langle H(Y), F(X, Y) Y\rangle+\langle H(Y), P(t, X, Y)\rangle+\frac{1}{\sqrt{n}}\langle H(Y), \operatorname{sgn} Y\rangle,
$$

so that by (3.1), (3.3) and (3.4)

$$
\begin{aligned}
& \dot{U} \leq-\left(\delta_{h} \delta_{f}\|Y\|-(a+1) \Delta_{h}\right)\|Y\|, \\
& \dot{U} \leq-1, \quad \text { if }\|Y\| \geq \max \left\{\frac{\Delta_{h}^{2}(a+1)^{2}+\delta_{h} \delta_{f}}{\delta_{h} \delta_{f} \Delta_{h}(a+1)}, L\right\}=D_{4} .
\end{aligned}
$$

Case (III). $\|X\| \geq 1$ and $\|Y\| \geq L$ follow from case (II) since $\dot{U}_{2}=0$ if $\|X\| \geq 1$ and $\|Y\| \geq L$. The two results (4.15) and (4.16) together imply that

$$
\dot{U} \leq-1 \text { provided }\|X\|^{2}+\|Y\|^{2} \geq D_{3}^{2}+D_{4}^{2} .
$$

This verifies (3.6) and Theorem 3.1 now follows.

Proof of Theorem 3.2. The procedure here is the same as that used for Theorem 3.1 but only that $P(t, X, Y) \neq 0$ as in the proof of Theorem 3.1. The proof of Theorem 3.2 is immediate as soon as we show (4.6) and (4.7). The verification of (4.6) given in $\S 4$ carries over with obvious modifications.

To verify (4.7), our starting point will be the estimates (4.8)-(4.14), which are still valid in this case. Thus, in obtaining estimates for $\dot{U}$ we shall consider points outside of the closed bounded set defined by $\|X\| \leq 1$ and $\|Y\| \leq L$. It will be convenient to 
consider the following three regions in turn: (I) $\|X\| \geq 1$ and $\|Y\| \leq L$, (II) $\|X\| \leq 1$ and $\|Y\| \geq L$, and (III) $\|X\| \geq 1$ and $\|Y\| \geq L$. For the case (I), we have from (4.8), (4.9) and (4.12) that

$$
\begin{aligned}
\dot{U}= & -\langle H(Y), F(X, Y) Y\rangle+\langle H(Y), P(t, X, Y)\rangle-\frac{\alpha}{L \sqrt{n}}\langle F(X, Y) Y, \operatorname{sgn} X\rangle \\
& -\frac{\alpha}{L \sqrt{n}}\langle G(X), \operatorname{sgn} X\rangle+\frac{\alpha}{L \sqrt{n}}\langle P(t, X, Y), \operatorname{sgn} X\rangle,
\end{aligned}
$$

so that by (3.1)-(3.3) and (3.7), and setting $\beta=\sqrt{n}$

$$
\dot{U} \leq-\frac{1}{L \beta}\left\{\alpha\langle G(X), \operatorname{sgn} X\rangle-\beta \mu L\left(1+\Delta_{h} L^{2}\right)\right\}+\Delta_{f},
$$

since $\|Y\| \leq L$. Thus, in view of (3.8), there exists a finite constant $D_{5}(>1)$, sufficiently large, such that $\dot{U} \leq-1$ provided $\|X\| \geq D_{5}$. As for the case (II): $\|X\| \leq 1$ and $\|Y\| \geq L$, we have from (4.8), (4.9) and (4.14) that

$$
\dot{U}=-\langle H(Y), F(X, Y) Y\rangle+\langle H(Y), P(t, X, Y)\rangle+\frac{1}{\sqrt{n}}\langle H(Y), \operatorname{sgn} Y\rangle,
$$

so that by (3.1), (3.3) and (3.7)

$$
\begin{aligned}
& \dot{U} \leq-\left(\left(\delta_{h} \delta_{f}-\mu \Delta_{h}\right)\|Y\|-\Delta_{h}\right)\|Y\|, \\
& \dot{U} \leq-1 \text { if }\|Y\| \geq \max \left\{\frac{\Delta_{h}^{2}+\left(\delta_{h} \delta_{f}-\mu \Delta_{h}\right)}{\Delta_{h}\left(\delta_{h} \delta_{f}-\mu \Delta_{h}\right)}, L\right\}=D_{6},
\end{aligned}
$$

where $\delta_{h} \delta_{f}-\mu \Delta_{h}>0$.

Case (III). $\|X\| \geq 1$ and $\|Y\| \geq L$, we have from (4.8), (4.9) and (4.14) that

$$
\dot{U}=-\langle H(Y), F(X, Y) Y\rangle+\langle H(Y), P(t, X, Y)\rangle
$$

so that by (3.1), (3.3) and (3.7) we obtain

$$
\dot{U} \leq-1 \text { if }\|Y\| \geq \max \left\{\left(\delta_{h} \delta_{f}-\mu \Delta_{h}\right)^{-\frac{1}{2}}, L\right\} .
$$

This verifies (3.6) and Theorem 3.2 now follows.

Next, we present an illustrative example to demonstrate the applicability of the results proved in this section.

Example 4.1. As a special case of (1.4), let us have for $n=2$ that

$$
\begin{gathered}
F(X, Y)=\left(\begin{array}{cc}
2+\frac{1}{x_{1}^{2}+y_{1}^{2}+1} & 1 \\
1 & 2+\frac{1}{x_{1}^{2}+y_{2}^{2}+1}
\end{array}\right), \quad G(X)=\left(\begin{array}{c}
2 x_{1}+\sin x_{1} \\
2 x_{2}+\sin x_{2}
\end{array}\right), \\
H(Y)=\left(\begin{array}{c}
y_{1}+\tan ^{-1} y_{1} \\
y_{2}+\tan ^{-1} y_{2}
\end{array}\right) \text { and } P(t, X, Y)=\left(\begin{array}{c}
\frac{1}{1+y_{1}^{2}}+\sin t \\
\exp ^{-x_{1}^{2}}
\end{array}\right) .
\end{gathered}
$$

Clearly, we have $\lambda_{1}(F(X, Y))=4-\sqrt{5}+\frac{1}{x_{1}^{2}+y_{1}^{2}+1}+\frac{1}{x_{2}^{2}+y_{2}^{2}+1}$ and $\lambda_{2}(F(X, Y))=$ $4+\sqrt{5}+\frac{1}{x_{1}^{2}+y_{1}^{2}+1}+\frac{1}{x_{2}^{2}+y_{2}^{2}+1}$. Thus, $4-\sqrt{5}<\lambda_{1}(F(X, Y)), \lambda_{2}(F(X, Y))<6+\sqrt{5}$, with $\delta_{f}=4-\sqrt{5}$ and $\Delta_{f}=6+\sqrt{5}$ 
It can easily be seen that

$$
J_{g}(X)=\left(\begin{array}{cc}
2+\cos x_{1} & 0 \\
0 & 2+\cos x_{2}
\end{array}\right),
$$

$\lambda_{1}\left(J_{g}\right)=2+\cos x_{1}, \lambda_{2}\left(J_{g}\right)=2+\cos x_{2}$, with $\delta_{g}=1$ and $\Delta_{g}=3$,

$$
J_{h}(Y)=\left(\begin{array}{cc}
1+\frac{1}{1+y_{1}^{2}} & 0 \\
0 & 1+\frac{1}{1+y_{2}^{2}}
\end{array}\right),
$$

$\lambda_{1}\left(J_{h}\right)=1+\frac{1}{1+y_{1}^{2}}, \lambda_{2}\left(J_{h}\right)=1+\frac{1}{1+y_{2}^{2}}$, with $\delta_{h}=1$ and $\Delta_{h}=2$, and lastly, it is obvious that vector $P(t, X, Y)$ above satisfies

$$
\|P(t, X, Y)\| \leq \sqrt{5} .
$$

It will be seen from the Figure 1 obtained by Maple 16, that the simulated solutions of the differential equation constructed are bounded. This further justifies our given results.

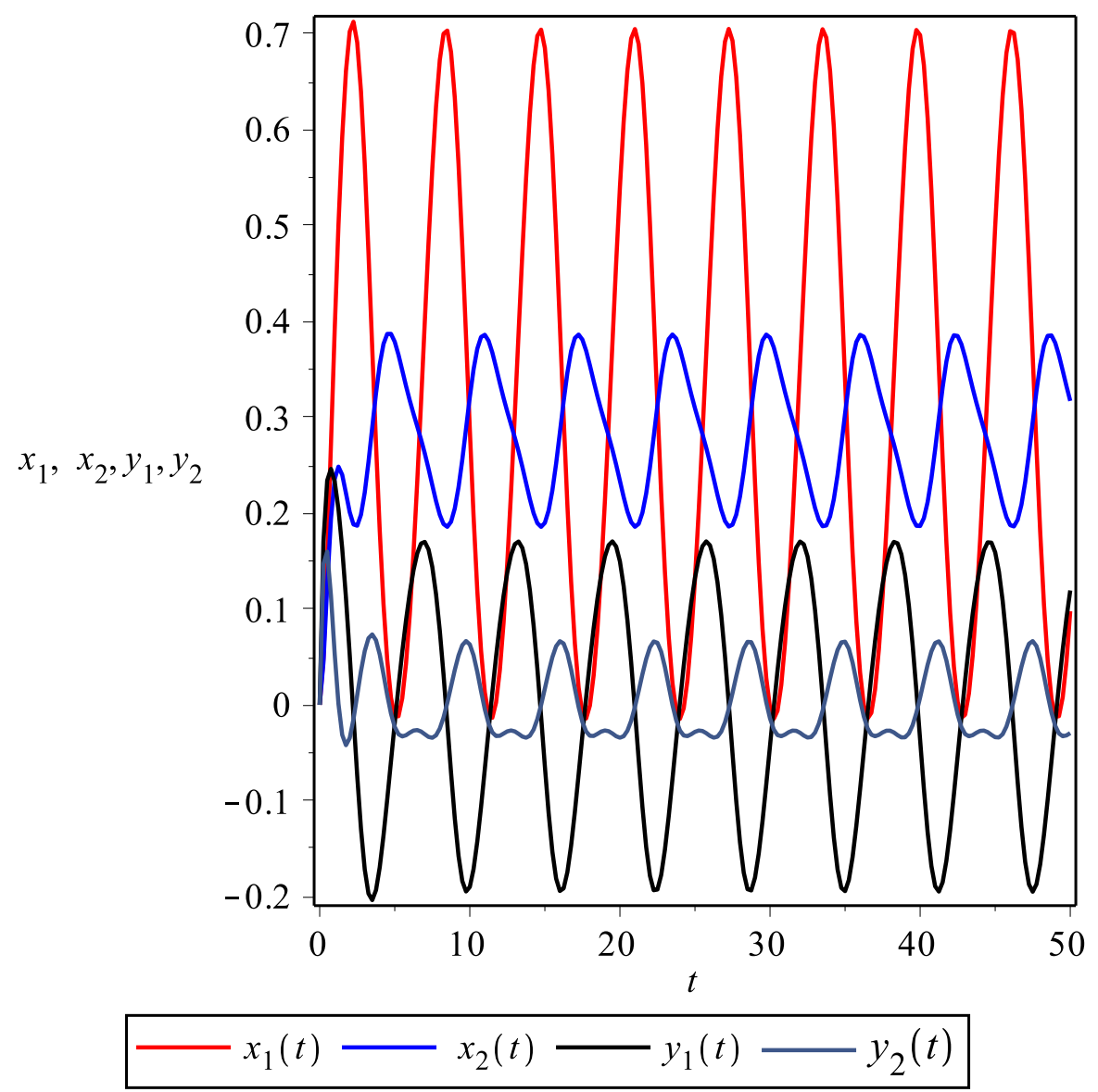

Figure 1. Solution paths of the given example. 


\section{REFERENCES}

[1] T. A. Ademola, M. O. Ogundiran, P. O. Arawomo and O. A. Adesina, Boundedness results for a certain third order nonlinear differential equations, Appl. Math. Comput. 216 (2010), 3044-3049.

[2] A. U. Afuwape, Ultimate boundedness results for a certain system of third-order non-linear differential equations, J. Math. Anal. Appl. 97 (1983), 140-150.

[3] A. U. Afuwape and M. O. Omeike, Further ultimate boundedness of solutions of some system of third order nonlinear ordinary differential equations, Acta Univ. Palack. Olomuc. Fac. Rerum Natur Math. 43 (2004), 7-20.

[4] E. N. Chukwu, On the boundedness of solutions of third-order differential equations, Ann. Mat. Pura Appl. 4 (1975), 123-149.

[5] J. O. C. Ezeilo and H. O. Tejumola, Boundedness and periodicity of solutions of a certain system of third-order non-linear differential equations, Ann. Mat. Pura Appl. 66 (1964), 283-316.

[6] J. O. C. Ezeilo and H. O. Tejumola, Further results for a system of third order ordinary differential equations, Atti Accad. Naz. Lincei Rend. Cl. Sci. Fis. Mat. Natur. 58 (1975) 143-151.

[7] F. W. Meng, Ultimate boundedness results for a certain system of third order nonlinear differential equations, J. Math. Anal. Appl. 177 (1993), 496-509.

[8] M. O. Omeike, O. O. Oyetunde and A. L. Olutimo, Boundedness of solutions of certain system of second-order ordinary differential equations, Acta Univ. Palack. Olomuc. Fac. Rerum. Natur. Math. 53 (2014), 107-115.

[9] M. R. Rao, Ordinary Differential Equations, Affiliated East-West Private Limited, London, 1980.

[10] R. Reissig, G. Sansone and R. Conti, Nonlinear Differential Equations of Higher Order, Noordhoff, Groninge, 1974.

[11] H. O. Tejumola, Boundedness criteria for solutions of some second-order differential equations, Accademia Nazionale Dei Lincei Serie VII 50(4) (1971), 204-209.

[12] H. O. Tejumola, Boundedness theorems for some systems of two differential equations, Atti. Accad. Naz. Lincei Rend. Cl. Sci. Fis. Mat. Natur. 51(6) (1971), 472-476.

[13] C. Tunc, On the stability and boundedness of solutions of nonlinear vector differential equations of third-order, Nonlinear Anal. 70(6) (2009), 2232-2236.

[14] C. Tunc, Boundedness of solutions of certain third-order nonlinear differential equations, J. Inequal. Appl. Math. 6(1) (2005), 1-6.

[15] C. Tunc and M. Atęs, Stability and boundedness results for solutions of certain third-order nonlinear vector differential equations, Nonlinear Dyn. 45(3-4) (2006), 273-281.

[16] T. Yoshizawa, Stability Theory by Lyapunov's Second Method, Publications of the Mathematical Society of Japan, Tokyo, 1966.

${ }^{1}$ Department of Mathematics,

Federal University of Agriculture, Abeokuta,

NigERIA

Email address: moomeike@yahoo.com

Email address: tjyanju2000@yahoo.com

Email address: danielogic2008@yahoo.com

${ }^{2}$ Department of Mathematics,

Lagos State University, Ojo,

NigERIA

Email address: aolutimo@yahoo.com 